\title{
A Model for Classification Secondary School Student Enrollment Approval Based on E-Learning Management System and E-Games
}

\author{
Hany Mohamed El-katary \\ College of Computing and \\ Information Technology, Arab \\ Academy for Science, Technology \& \\ Maritime Transport, Cairo, Egypt
}

\author{
Essam M. Ramzy Hamed \\ College of Computing and \\ Information Technology, Arab \\ Academy for Science, Technology \& \\ Maritime Transport, Cairo, Egypt
}

\author{
Safaa Sayed Mahmoud \\ Ain Shams University \\ Cairo, Egypt
}

\begin{abstract}
Student is the key of the educational process, where students' creativity and interactions are strongly encouraged. There are many tools embedded in Learning Management Systems (LMS) that considered as a goal evaluation of learners. A problem that currently appeared is that assessment process is not always fair or accurate in classifying students according to accumulated knowledge. Therefore, there is a need to apply a new model for better decision making for students' enrollment and assessments. The proposed model may run along with an assessment tool within a LMS. The proposed model performs analysis and obtains knowledge regarding the classification capability of the assessment process. It offers knowledge for course managers regarding the course materials, quizzes, activities and e-games. The proposed model is an accurate assessment tool and thus better classification among learners. The proposed model was developed for learning management systems, which are commonly used in e-learning in Egyptian language schools. The proposed model demonstrated good accuracy compared to real sample data (250 students).
\end{abstract}

Keyword-evaluation; learning management system; e-games; classification

\section{INTRODUCTION}

Learning process goes through many generations starting from traditional learning till electronic learning. Traditional learning would be the oldest and the first process of learning then distance learning (DL) and finally electronic learning appears which would be the earliest educational phase process. Traditional learning used for centuries it is based mainly on face to face learning where a lecturer and a group of students meet with each other at certain place.

DL has various ways of descriptions with the more popular formats such as audio, video, broadcast radio and television. DL is characterized by the separation of geographic distance and time difference. E-learning is the use of internet and digital technologies to create experiences that educate the follow of human beings.

Learning Management Systems (LMSs), which is one of the E-learning tools, provide a wide set of functionalities to support students' learning such as file storage, forums, calendar, news, mail, submission management system, groups surveys , organization, assessments, FAQs (Frequently Asked Questions) or scheduling and educational games. All these types of education have different ways of learning but evaluation process become one of the big challenges for classifying students in fair enrollment approval based on new educational technologies as LMS.

Sotiris, Athanasios and Savvas, mentioned some advantages of LMS in [1] which are:

- If a pupil loses a tutorial because of illness or participation in school activities, he/she has the ability to have access to the presentations, the examples and all the teaching material.

- The pupils have better assimilation of the course concepts in comparison to the ones of previous years, since they can do exercises and tests from their home and evaluate their knowledge.

- The pupils recognize that computers do not exist only for playing games but also as a mean to gain knowledge. Since they are familiar with LMS, they will probably correspond very easily later, in the requirements of their academic studies.

Although there are many successful LMS systems, Sabine ,Kinshuk and Tzu-Chien Liu [2] , concluded in their paper that matching students with learning material and activities Which may fit their preferred ways of learning and study can make learning easier for them. This matching hypothesis is supported by educational theories. The characteristics of each type of student is initiated upon his behavior for example sensing learners like solving problems based on standard procedures.

Classifying student upon his behavior is the main conclusion of this paper.

Yücel urlu, Dai Hasegawa, Hiroshi Sakuta [3] tried to discover a relation between student and LMS by considering the characteristics of the students in order to understand their needs. Student access rates were correlated with their needs, interests, and personal motivations.

Dave Moursund mentioned the importance of educational games for both student and teacher. Using crossword puzzles can help in maintaining and improving vocabulary, spelling skills and knowledge of many miscellaneous tidbits of information [4]. 
This paper mentioned through results that e-learning and educational games learning was clearly better used in education process rather than traditional learning, the new designed model was more accurate in classifying students based on their interests using WEKA as a data mining tool.

In this paper, related work are explained in section II. In section III, the discussion of the proposed model and the implementation of the proposed model. In section IV results of ten classification algorithms including their performance measures. Comparative analysis, and conclusions are explained in sections $\mathrm{V}$ and VI respectively.

\section{RELATED WORK}

Waraporn Jirapanthong [5] designed a new model which can support students in Thailand to choose their courses based on number of factors as input sector, weight of vector and total number of neuron and applying number of classification algorithm to complete this process.

Qing Yang1, Junli Sun1, Jinqiao Wang1[6] implementing system which created an ontology file for classifying students upon their interest by calculating the similarity of different users by the using of the relationship between the concepts in domain ontology.

Jili Chen, Feng Wang, Kebin Huang, Huixia Wang [7] proposed a new method of classifying student behavior by measuring different student activities by using Fuzzy clustering method to mine E-learning behavior patterns using browsing behavior with Web pages and other learning resources. The learner's behavior can be perceived by clicking on a link, staying at a page.

Andrea, Marco [8] designed a system SOCIALX which is a web application. This system classifies students into 6 classes, first: Involvement which student can be measured by number of contributions that submitted by any student or grades given or acceptability. Second: usefulness students which measures how students contribute others. Third: competence: which measure complains from students and teachers. Fourth: judgment which can measure the ability of student judgment others. Fifth: self-judgment: which measure how student be fair with himself related to teacher evaluation. Sixth: active critical system: which measure the creativity of that student.

Yücel urlu, Dai Hasegawa, Hiroshi Sakuta [9] divided students' topics and count number of accessing for each topic for each student to measure the highest for each student. Also they concluded the highest accessed material which student access it, they concluded that e-learning systems can be used to improve student-learning patterns and help us in improving traditional courses as well as e-Learning systems.

\section{THE PROPOSED MODEL}

The proposed approach, with its main features is essentially based on two components: the student phase and the evaluation phase. Figure1 shows a block diagram for the components of the proposed model used.

\section{A. Model Architecture}

This diagram consists of two basic levels or phases, the first one is student phase and the second one is the evaluation phase. In student phase gathering information is done rather automatically based on the online behavior and activities of students as registration ,notification ,course documents , interface tutorial, announcements, useful links , student papers , exercises, quizzes and semantic search which the model can extract information from all these activities.

Huge amount of data are collected continuously from the student interactions with materials, exams and educational games as illustrated there are different resources embedded in the LMS systems which in this model can extract information from students for the next evaluation phase. In evaluation phase student interests gathered and evaluated by comparing each class of study and take the decision for the dedicated student class of interest.

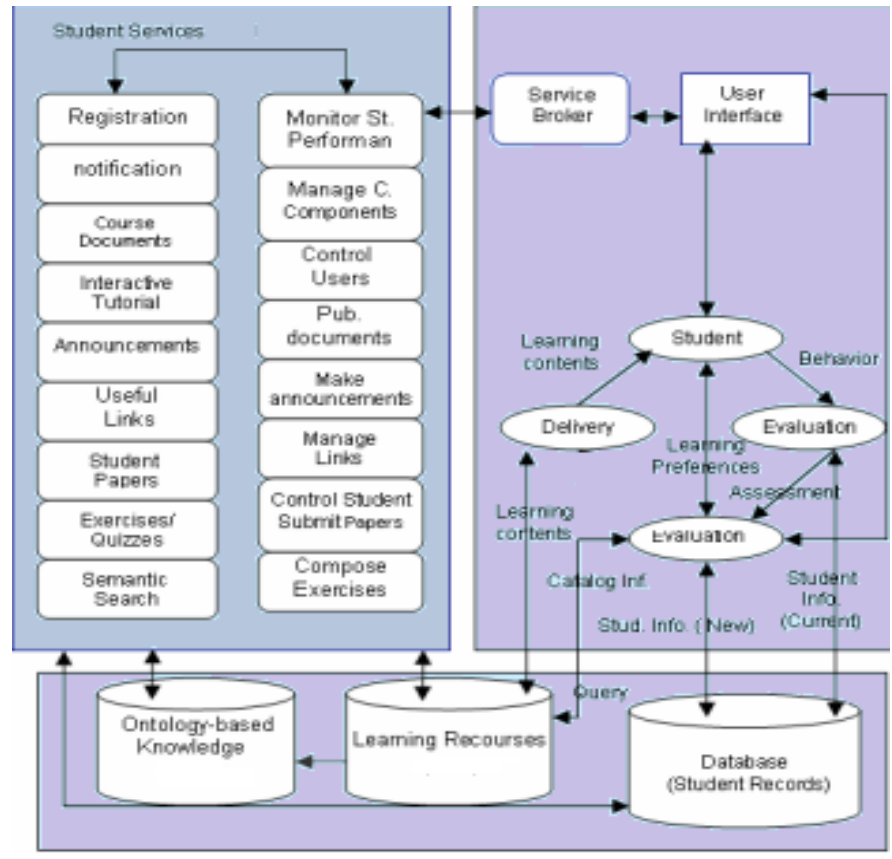

Fig. 1. The proposed model Architecture

\section{B. Methodology of the proposed model and implementation}

To implement and evaluate the proposed approach, a conceived system composed by a set of components, where each component is performing a number of student activities. The main features of the proposed recommender system are shown in the next paragraph.

This system provides an analysis of the attributes. Which can trace the distribution of students within each section according to their courses, their activities and educational games desire when achieving school section criteria, which include Grades Qualified Materials.

At this study divided student evaluation into two stages first stage for classifying students, either his interest is scientific or literary, second stage to classify scientific students either, science scientific interest students or mathematical scientific interest students.

As Sebastian Arnold, Jun Fujima, Andreas Karsten and Harald Simeit [10] designed a new model based on game theory which can be adapted for each learner upon his own 
preferences which divided into four classes each class can meet with every learner behavior.

Evaluation process starts with filling basic information for each student as his name and sex and birthdate and academic School year as illustrate in fig 2 .then second screen appears for gathering courses, activities and e-games grades. Each material classified previously as scientific material or literary material. Gathering material starting from KG1 till

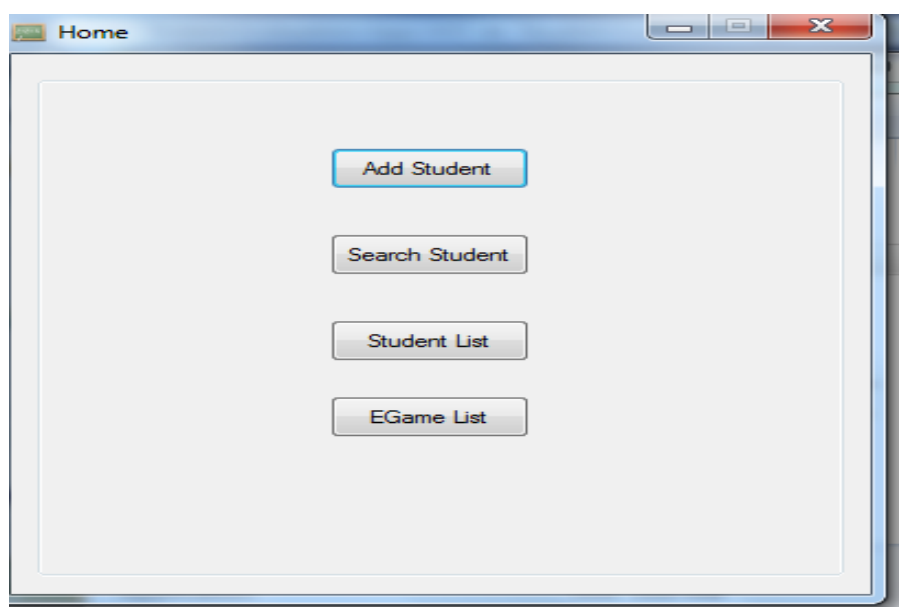

Fig. 2. Main Menu for student evaluation model

Year 11 and every academic year have its own evaluation result. Last year evaluation (Y11) depends on the accumulative previous years evaluations. Ontology based knowledge set the rules and relations between material, activities and e-games for the same section so model can decide either this material or activity or e-game is scientific or literary. Actually system didn't allowed to extract last year evaluation which is Y11 until evaluated the last 4 years (Y7, Y8, Y9 AND Y10) which called first evaluation process as illustrated in fig.3 which shows students summation for each year at each section and the decision taken based on the comparison between each section to choose the highest which represent student interest. Then go through second evaluation as showed in fig. 4 which is on Y11 with its final result for this student. Material used for this classification for each section was as follow:

Literary section $=$ (Geography, History, and Arabic).Science section which is divided into two sub sections which are:

- Scientific science section $=($ Physics, chemistry and biology).

- Mathematical science section $=$ (Geometry and Algebra).

These materials including student activities, grades and educational games which model compare the sum for each section and take the highest.

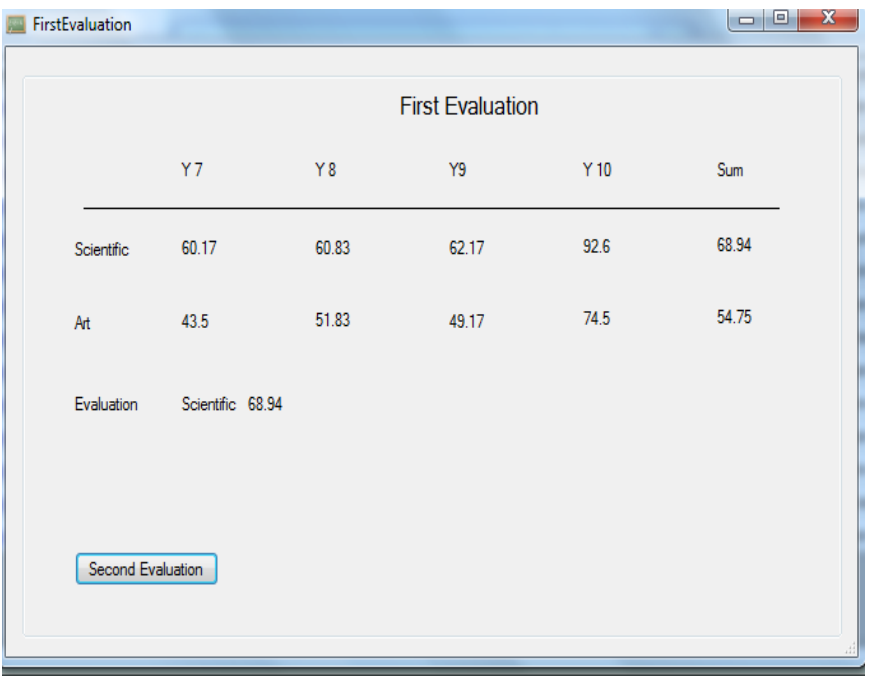

Fig. 3. First student screen with major student behavior evaluation

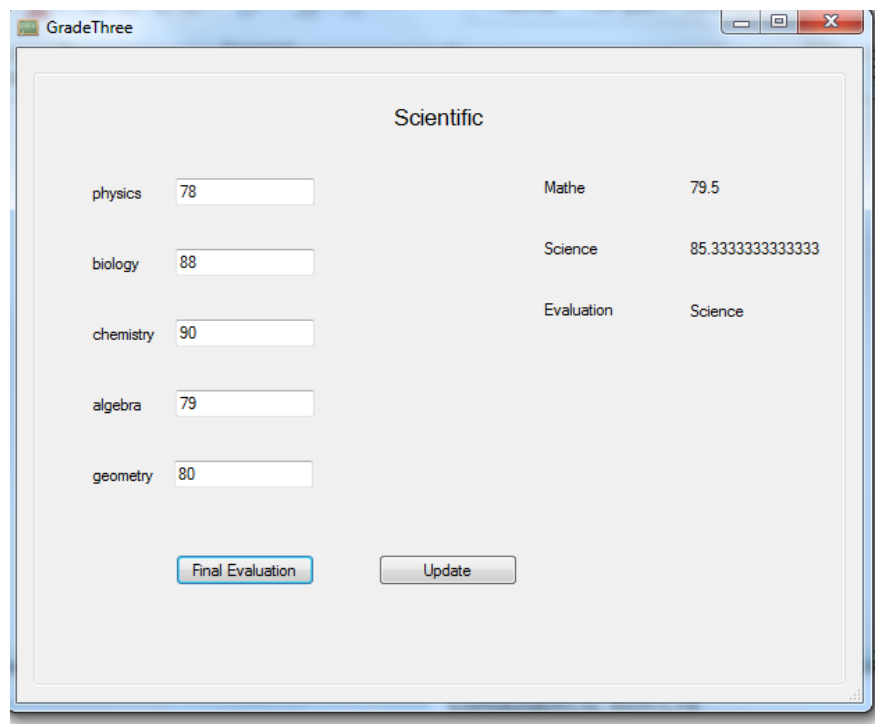

Fig. 4. Second student evaluation screen with minor student behavior evaluation ( Science or Math)

\section{Results Methodology}

Figure 5 illustrate the steps after collecting data from implemented model and feeding it to WEKA as a miming tool. After gathering student data either online or offline, this data get cleaned and preprocess to convert it to .ARFF file as WEKA can deal with this kind of files, then applying 10 different kinds of classification algorithms for mining students data. Finally calculate accuracy to choose the best. 


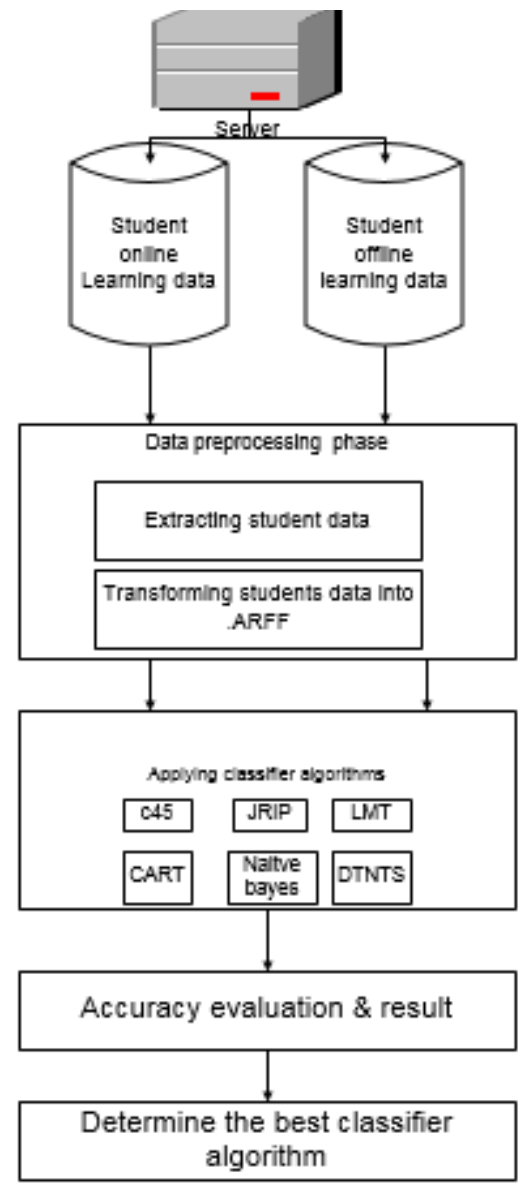

Steps for the proposed students - Model

Fig. 5. Data Collection from students Mining Procedure

\section{EXPERIMENTAL RESULTS}

The previous section described the patterns which are incorporated for each dimension as well as whether a high or low occurrence indicates a specific learning section preference. Based on this information, data about students' behavior can be used to calculate hints for specific learning section preferences. For example, if a learner often visited LMS courses or activities or games, this gives us a hint that the learner attend otherwise it gives us hints that this student is not a regular student for LMS.

There are many patterns which clarify the importance of using LMS than traditional learning such as attendance time of access.

Cavus, Uzunboylu and Ibrahim [11] underlined that a learning management system (LMS) provides the platform for web-based learning environment by enabling the management, delivery, tracking of learning, testing, communication, registration process and scheduling.

Fig.6 shows the importance of using LMS than using traditional learning and verified the advantages for using LMS as mentioned previously.
Figure 6 illustrate a comparison between different types of learning based on the access time duration (hours / month) for the three courses chosen which are Math, Science and English. Main major notification was that students prefer using LMS and E-games than traditional learning also minor notification was the number of students increased in later months in using LMS system and E-games than traditional learning. So, the traditional learning comes at third priority after LMS and Egames learning.

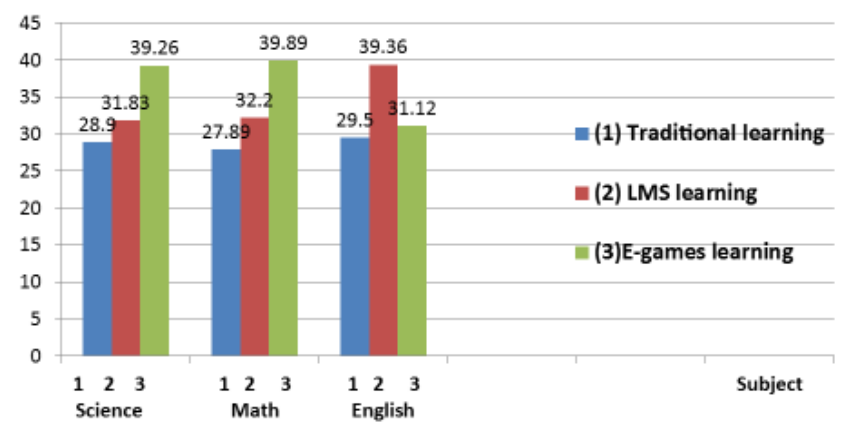

Fig. 6. Science, Math and English courses comparision using number of access time duration (hours/month) for learning types

Classification is one of the data mining techniques that is mainly used to analyze a given dataset and takes each instance of it and assigns this instance to a particular class with the aim of achieving least classification error. It is used to extract models that correctly define important data classes within the given dataset. It is a two-step process. In first step the model is created by applying classification algorithm on training data set.

Then in second step, the extracted model is tested against a predefined test dataset to measure the model trained performance and accuracy. So, classification is the process to assign class label for this dataset whose class label is unknown. Versatile list of techniques are available for classification like decision tree induction, Bayesian classification, and Bayesian network.

Figure 7 showes results for TP, FP, Precesion and recall after using WEKA for classifying students for scientific class using 10 different types of algorithms which mostly used in educational field [12].

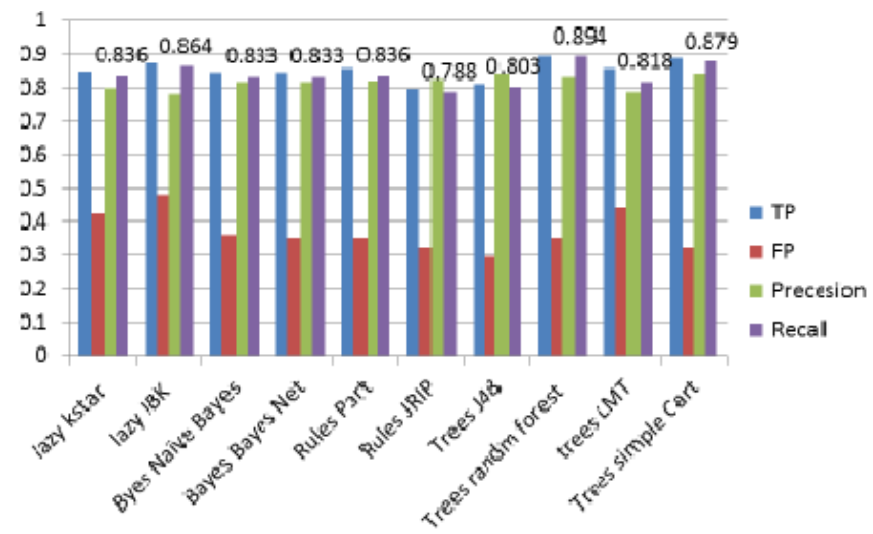

Fig. 7. Different parametes for 10 algorithms for E-game for science class 
Results show that TREE RANDOM FOREST gives the best performance, then CART, LAZY IBK, LAZY K-STAR, RULES PART, Byes Naïve Bayes, Bayes Bayes Net, TREE LMT respectively. The TREE J48 algorithm comes in the Ninth place and Rules JRIP come at the end of the order.

Fig. 8 showes results for TP, FP, Precesion and recall after using WEKA for classifying students for literary class using 10 different types of algorithms which mostly used in educational field.

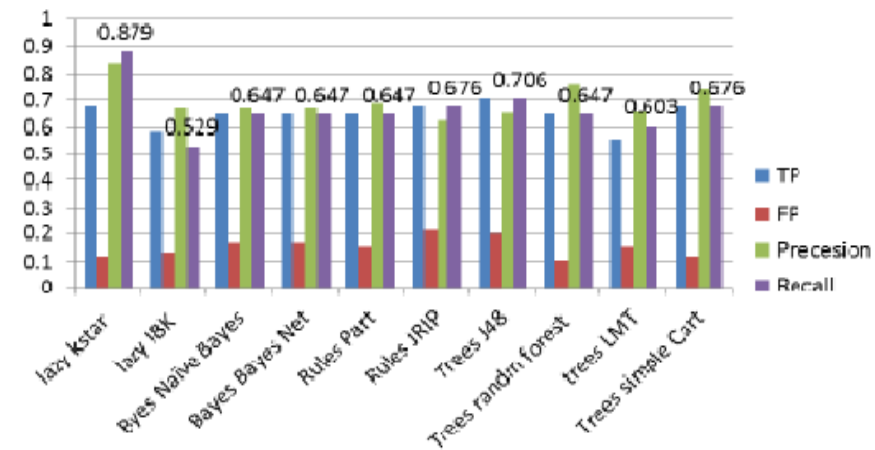

Fig. 8. Different parametes for 10 algorithms for E-game for Literary class

Results show that lazy k-star gives the best performance, then TREE J48, RULES JRIP, TREES SIMPLE CART, Byes Naïve Bayes, Bayes Bayes Net and tree random forest, rules part the same performance, respectively. The Tree LMT algorithm come in the ninth place and lazy IBK come at the end of the order.

\section{COMPARATIVE ANALYSIS}

When evaluating students $(\mathrm{N}=250)$ which was collected from national language school in Egypt from different level of education as the sample shown in Fig.9 through the model and comparing the result deduced from the system for Y11 (second secondary school) with the data collected from result of Y12 (third secondary school) at their school. Conclusion in Table I shows the classification of each section and the percentage of each. Fig.10 displays final comparison between three types of learning included in this study which are LMS , E-games and Traditional learning, which shows that LMS has 85\% accuracy (Rules Part Algorithm), E-games has 82\% accuracy (Tree Random Forest Algorithm) and finally traditional learning has $80.4 \%(49 / 250=19.6 \%, 100-19.6=80.4 \%)$.

\begin{tabular}{|c|c|c|c|c|c|c|c|c|c|}
\hline S.ID & Geography & History & Arabic & Philosophy & Physics & Geometry & Algebray & Chemistry \\
\hline 10001 & 90 & 77 & 73 & 81 & 85 & 83 & 92 & 91 \\
\hline 10002 & 89 & 80 & 78 & 82 & 90 & 90 & 80 & 93 \\
\hline 10003 & 89 & 78 & 80 & 85 & 85 & 90 & 91 & 92 \\
\hline 10004 & 79 & 88 & 91 & 79 & 89 & 86 & 89 & 79 \\
\hline 10005 & 89 & 69 & 74 & 85 & 87 & 90 & 91 & 92 \\
\hline 10006 & 91 & 76 & 72 & 80 & 84 & 82 & 91 & 90 \\
\hline 10007 & 69 & 80 & 77 & 68 & 80 & 90 & 72 & 88 \\
\hline 10008 & 92 & 79 & 79 & 83 & 86 & 87 & 90 & 92 \\
\hline 10009 & 80 & 88 & 90 & 78 & 79 & 90 & 87 & 90 \\
\hline 10010 & 91 & 88 & 79 & 80 & 79 & 78 & 87 & 89 \\
\hline
\end{tabular}

Fig. 9. Data set sample according to their grades

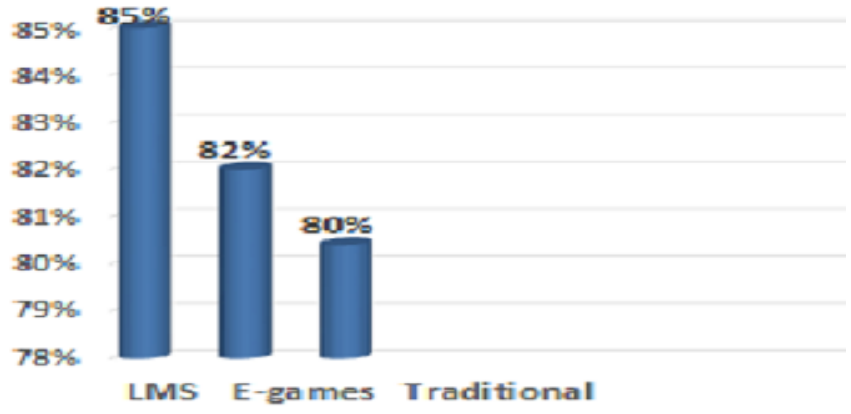

Fig. 10. Accuracy results for three types of learning in evaluation enrollment study

The results for each branch is calculated by the next equation.

Branch Success rate $=$ number of succeeded students in section / total number of students at this section.

Math success rate $=(71 / 76) * 100=93.4 \%$

Science success rate $=(69 / 73) * 100=94.5 \%$

Literary success rate $=(90 / 101) * 100=89.1 \%$

And the total average success for all the recommender system $=(($ Math success rate + science success rate + literary rate) / 3) * 100 .

The success average percentage of the recommender system $=((93.4+94.5+89.1) / 3) * 100=92.3 \%$

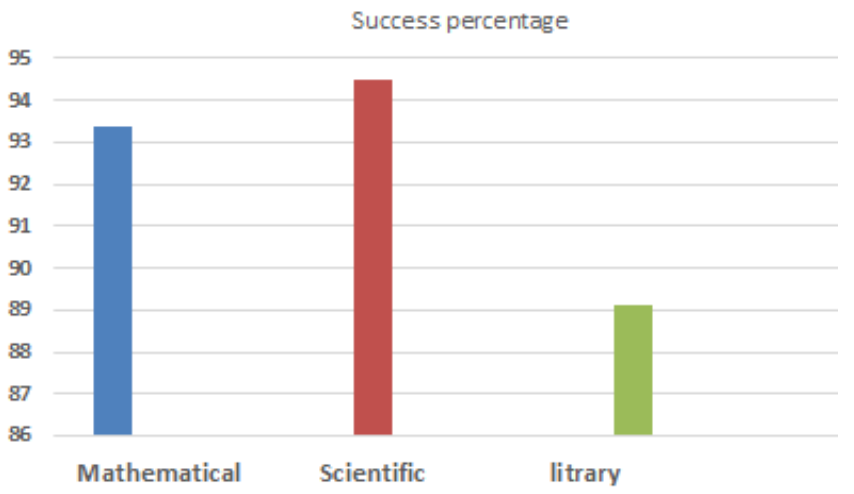

Fig. 11. System success for each branch

On the other hand real data gathered for students for Y12 was calculated as next equation:

Branch Success rate $=$ number of succeeded students in section / total number of students at this section.

So, Math success rate $=(76 / 85) * 100=89.4 \%$,

and Science success rate $=(65 / 71) * 100=91.5 \%$,

and Literary success rate $=(83 / 94) * 100=88.2 \%$

Then, the total average success for all the recommender system $=(($ Math success rate + science success rate + literary rate) / 3) $* 100$.

So, the success average percentage of the real data $=((89.4$ $+91.5+88.2) / 3) * 100=89.7 \%$ 


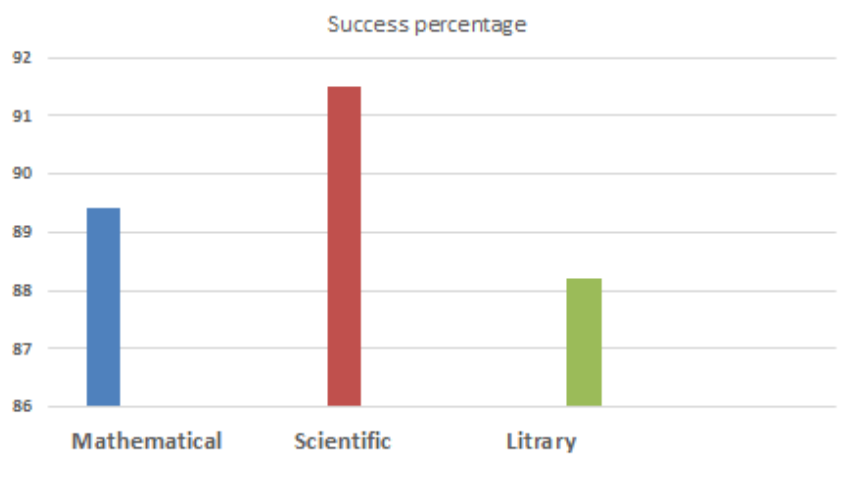

Fig. 12. Real success data for each branch

\section{CONCLUSIONS AND FUTURE WORK}

This work introduced an automatic student modeling approach for identifying learning skills based on LMS. The proposed model used behavior of students during they are learning in order to gather hints about their learning skills. By applying a simple rule-based mechanism, learning skills are calculated based on the gathered indications. By comparing different types of materials as online courses, online activities, and online educational games, this work can deduce the most suitable section which students can specialize on it. Improving educational games would be one of the main points for improving educational process. Compared with statistical analysis methods, this model is more effective, the process is more intelligent, and the result is more accurate. It shows that by using suggested model, teachers can understand the students better in interest, material and other information. Educational games not only improve educational process but also improve evaluation process through calculating different parameters which will be used in the future as access duration, material type and access level. The evaluation of the approach demonstrated good results and showed that the approach is suitable for identifying learning skills with respect to the new model.

Future research should include multiple schools and examine differences based on region, available resources. Future research could also be done to include undergraduate students and compare the perceptions of undergraduate, graduate students, institute students and faculty.

\section{REFERENCES}

[1] Sotiris Manitsaris, Athanasios Perdos, Savvas Pavlidis, "An open source Learning Management System (ASDL) using ICT for High Schools", Proceedings of the Sixth International Conference on Advanced Learning Technologies , 2006 IEEE, Macedonia Universty, Thessaloniki.

[2] Sabine Graf, Kinshuk, Tzu-Chien Liu , Identifying Learning Styles in Learning Management Systems by Using Indications from Students' Behavior, Eighth IEEE International Conference on Advanced Learning Technologies, July 2008, PP 482 - 486, Santander, Cantabria.

[3] Yücel urlu, Dai Hasegawa, Hiroshi Sakuta, Student Interactions with Elearning Systems: User and Topic Analysis, 3-5April 2014., Military Museum and Cultural Center, Harbiye, Istanbul, Turkey.

[4] Dave Moursund, "Introduction to Using Games in Education: A Guide for Teachers and Parents" Teacher Education, College of Education University of Oregon 97403, 2006.

[5] Waraporn Jirapanthong , Classification Model for Selecting Undergraduate Programs , 2009 Eighth International Symposium on Natural Language Processing, IEEE, Bangkok, Thailand, pp. 89-95.

[6] Qing Yang1, Junli Sun1, Jinqiao Wang1, Semantic Web-Based Personalized Recommendation System of Courses Knowledge Research, 2010 International Conference on Intelligent Computing and Cognitive Informatics.

[7] Jili Chen, Feng Wang, Kebin Huang, Huixia Wang , E-learning Behavior Analysis based on Fuzzy Clustering , 2009 Third International Conference on Genetic and Evolutionary Computing.

[8] Andrea Sterbini , Marco Temperini, "Social Exchange and Collaboration in a Reputation-Based Educational System", Information Technology Based Higher Education and Training, 2010 9th International conference, IEEE, PP 201-207.

[9] Yücel urlu, Dai Hasegawa, Hiroshi Sakuta , Student Interactionz with E-learning Systems: User and Topic Analysis, 2014 IEEE Global Engineering Education Conference (EDUCON).

[10] Sebastian Arnold*, Jun Fujima*, Andreas Karsten $\dagger$ and Harald Simeit $\dagger$, Adaptive Behavior with User Modeling and Storyboarding in Serious Games, 2013 International Conference on Signal-Image Technology \& Internet-Based Systems.

[11] Cavus, N., Uzunboylu, H., \& Ibrahim, D. (2007). Assessing the success of students using a learning management system and together with a collaborative tool in Web-based teaching of programming languages. Journal of Educational Computing Research, Vol. 36(3) 301-321, 2007.

[12] Abdul Hamid M. Ragab, Amin Y. Noaman, Abdullah S. AL- Ghamd, Ayman. Madbouly," a comparative Analysis of Classification Algorithms for Students College Enrollment Approval Using Data Mining”, IDEE 14 Proceedings of the 2014 Workshop on Interaction Design in Educational Environments, ACM International Conference Processing Series. 